\title{
BMJ Open Relationship between sociodemographics, healthcare providers' competence and healthcare access among two-spirit, gay, bisexual, queer and other men who have sex with men in Manitoba: results from a community-based cross-sectional study
}

\author{
Rusty Souleymanov (D) , ${ }^{1}$ Jared Star (1) , ${ }^{2}$ Albert McLeod, ${ }^{3}$ Sana Amjad, ${ }^{2}$ \\ Samantha Moore, ${ }^{2}$ Christopher Campbell, ${ }^{4}$ Robert Lorway, ${ }^{2}$ Michael Payne, ${ }^{5}$ \\ Laurie Ringaert, ${ }^{6}$ Linda Larcombe, ${ }^{7}$ Gayle Restall, ${ }^{8}$ Paula Migliardi, ${ }^{9}$ \\ Bryan Magwood, ${ }^{10}$ Nathan J Lachowsky (D) , ${ }^{11,12}$ David J Brennan (D) , ${ }^{13}$ \\ Uday Norbert Sharma ${ }^{1}$
}

To cite: Souleymanov R, Star J, McLeod A, et al. Relationship between sociodemographics, healthcare providers' competence and healthcare access among two-spirit, gay, bisexual, queer and other men who have sex with men in Manitoba: results from a community-based crosssectional study. BMJ Open 2022;12:e054596. doi:10.1136/ bmjopen-2021-054596

- Prepublication history for this paper is available online. To view these files, please visit the journal online (http://dx.doi org/10.1136/bmjopen-2021054596).

Received 16 June 2021 Accepted 05 January 2022

Check for updates

(c) Author(s) (or their employer(s)) 2022. Re-use permitted under CC BY-NC. No commercial re-use. See rights and permissions. Published by BMJ.

For numbered affiliations see end of article.

\section{Correspondence to} Dr Rusty Souleymanov; Rusty.Souleymanov@umanitoba. ca

\section{ABSTRACT}

Objective Little is known about barriers to healthcare access for two-spirit, gay, bisexual and queer (2SGBQ+) men in Manitoba.

Design Data were drawn from a community-based, cross-sectional survey designed to examine health and healthcare access among $2 \mathrm{SGBQ}+$ men.

Setting Community-based cross-sectional study in Manitoba, Canada.

Participants Community-based sample of 368 2SGBQ+ men.

Outcomes Logistic regression analyses assessed the relationship between sociodemographics, healthcare discrimination, perceived healthcare providers' $2 \mathrm{SGBQ}+$ competence/knowledge and two indicators of healthcare access (analytic outcome variables): (1) having a regular healthcare provider and (2) having had a healthcare visit in the past 12 months.

Results In multivariate analyses, living in Brandon (adjusted OR (AOR) $=0.08,95 \% \mathrm{Cl} 0.03$ to 0.22 ), small cities ( $A O R=0.20,95 \% \mathrm{Cl} 0.04$ to 0.98 ) and smaller towns $(\mathrm{AOR}=0.26,95 \% \mathrm{Cl} 0.0800 .81$ ) in Manitoba (compared with living in Winnipeg), as well as having a healthcare provider with poor (AOR=0.19, 95\% $\mathrm{Cl} 0.04$ to 0.90 ) or very poor competence/knowledge $(\mathrm{AOR}=0.03,95 \% \mathrm{Cl}$ 0.03 to 0.25 ) of $2 S G B Q+$ men's issues (compared with very good competence) was associated with lower odds of having a regular healthcare provider. Living in Brandon ( $\mathrm{AOR}=0.05,95 \% \mathrm{Cl} 0.02$ to 0.17 ) and smaller towns (AOR $=0.25,95 \% \mathrm{Cl} 0.67$ to 0.90 ) in Manitoba (compared with living in Winnipeg) was associated with lower odds of having a healthcare visit in the past 12 months, while identifying as a gay man compared with bisexual (AOR $=12.57,95 \% \mathrm{Cl} 1.88$ to 83.97 ) was associated with higher odds of having a healthcare visit in the past 12 months.

Conclusions These findings underscore the importance of reducing the gap between the healthcare access of

\section{Strengths and limitations of this study}

- To our knowledge, this is the first study of two-spirit, gay, bisexual and queer $(2 \mathrm{SGBQ}+)$ men in Manitoba, Canada.

- We used logistic regression analysis to examine the association between sociodemographics, discrimination in healthcare, perceived health practitioner's competence regarding issues affecting $2 \mathrm{SGBQ}+$ men and healthcare access among a sample of 368 $2 S G B Q+$ men in Manitoba.

- In terms of limitations, the data relied on crosssection survey design, which presents issues with establishing directionality of findings.

rural and urban $2 \mathrm{SGBQ}+$ men, improving healthcare providers' cultural competence and addressing their lack of knowledge of $2 \mathrm{SGBQ}+$ men's issues.

\section{INTRODUCTION}

Cisgender and transgender two-spirit, gay, bisexual, queer (2SGBQ+) and other men who have sex with men in Manitoba (one of Canada's Prairie provinces) remain vulnerable populations for a variety of health disparities. Very limited research is available about 2SGBQ+ men's healthcare access in Manitoba. To supplement this gap in knowledge, this study examined sociodemographic and socioecological factors associated with healthcare access among $2 \mathrm{SGBQ}+$ men.

$2 \mathrm{SGBQ}+$ men disproportionately experience poorer levels of physical, sexual and mental health, as well as significant barriers to 
healthcare access. ${ }^{1-22}$ Both Canadian ${ }^{7-9} 2324$ and international research (mainly conducted in the USA) ${ }^{25-28}$ have shown that experiences of marginalisation and discrimination are associated with negative health outcomes and healthcare access.

Research on healthcare access among two-spirit, lesbian, gay, bisexual, transgender, queer, intersex and asexual (2SLGBTQIA+) and other sexual and gender minority populations suggests that these communities have unique health needs that may not be met by existing healthcare services. ${ }^{29}{ }^{30}$ Some of these barriers include healthcare professionals' lack of competence and knowledge of 2SLGBTQIA+ persons' healthcare needs, as well as negative attitudes towards 2SLGBTQIA+ people. ${ }^{31-34}$ 2SLGBTQIA+ peoplemay also be reluctant to disclose their sexual orientation, which may lead to them receiving inadequate care. ${ }^{33} 34$

When it comes to 2SGBQ+ men, research demonstrates that they also experience systematic marginalisation and discrimination in health and social care contexts. ${ }^{135-40}$ Many 2SGBQ+ menwill avoid healthcare settings they do not feel safe in and are far more likely to access 2SGBQ-specific services for support with their healthcare needs. ${ }^{35-40} 2 \mathrm{SGBQ}+$ men are also more likely than heterosexual men to consult a family doctor and mental health provider, report unmet healthcare needs and experience sexual orientation stigma within primary care. ${ }^{41-44}$

Furthermore, healthcare access may be affected by 2SGBQ+ men's sociodemographic and socioecological factors, including education and income level, ethnicity, pre-existing health status or geographic locations ${ }^{137-39} 45$ With regards to geography, most studies of $2 \mathrm{SGBQ}+$ men have historically been conducted in urban centres due to ease of access to high concentrations of these populations in urban settings. Research suggests that rural areas may be less hospitable to 2SLGBTQIA+ people, due to increased stigma and social isolation in rural areas. ${ }^{46}$ While disclosure of sexual orientation or identity in rural communities may increase access to 2SLGBTQIA+ appropriate social support and healthcare, this may also increase the risk of discrimination and stigma. ${ }^{46}$

Other barriers to care documented in the research include the lack of advertisement of sexuality and gender-affirming providers, gaps in coverage, cost-related hurdles, poor treatment from healthcare providers, as well as a lack of competency and the presence of negative attitudes from healthcare providers related to serving this population. ${ }^{478}$ Understanding these barriers and the additional health risks they impose is crucial to improving the health and well-being of $2 \mathrm{SGBQ}+$ men. The distinct use of or access to services documented in Canadian and international literature indicates the need for a closer examination of the experiences of Manitoban 2SGBQ+ men when it comes to healthcare access.

\section{Indicators of healthcare access}

Access to a healthcare provider is commonly measured by assessing whether an individual has had a healthcare visit in the past 12 months. ${ }^{38}$ Research from the USA suggests that $27 \%$ of gay male adults report either no annual visits or a minimum of one instance of healthcare access. ${ }^{38}$ However, using annual visits as an indicator of healthcare access for $2 \mathrm{SGBQ}+$ men in Manitoba is limited, as this measure does not take into account whether the individual has one provider they consider their regular source of healthcare. Having one provider that acts as a regular care provider may be a more useful indicator to healthcare access, as this definition implies both continuity and coordination of primary care..$^{88} 490$ Therefore, access to regular healthcare providers is another component of healthcare access. Research consistently shows that having access to a healthcare provider or access to regular healthcare facilities where individuals can receive regular care is associated with better health outcomes, fewer health disparities and lower healthcare costs. ${ }^{51}$ Therefore, this study assessed two indicators of healthcare access for $2 \mathrm{SGBQ}+$ men, including their access to regular healthcare providers and their visits to healthcare providers in the past 12 months.

\section{Theoretical frameworks}

This study approaches the healthcare access of 2SGBQ+ men in Manitoba as a multilevel challenge, requiring an understanding of a variety of factors at individual, social and structural levels. ${ }^{4}$ Socioecological systems theory ${ }^{5354}$ provides a framework to examine this population's healthcare access within the context of individual and socio-structural levels. This project was informed by socioecological systems theories, ${ }^{53}$ which considers that different factors (geography, sexual orientation identity and service providers' competence) may operate at multiple levels (individual, interpersonal and structural) to impact this population's healthcare access. A socioecological approach recognises the interdependence of people and their environment. ${ }^{53}$ Social ecological approaches to healthcare access and outcomes explore individual level factors, including practices, interpersonal relationships and knowledge, and structural level factors that include healthcare environments. ${ }^{51} 55$ Geographic location is an example of a sociodemographic characteristic associated with access to a regular source of healthcare, specifically, research conducted in global contexts showing that LGBT people living in proximity to urban centres are more likely to report better healthcare access. ${ }^{51}$ Our theoretical lens informed our methods, the selection of variables for investigation in this project and the holistic approach to conceptualisation of healthcare access.

\section{The current study}

Previous research has established a relationship between healthcare access and discrimination, primarily among $2 \mathrm{SGBQ}+$ men in other Canadian provinces. ${ }^{139101744}$ Similarly, other studies have identified sociodemographics and discrimination as potentially important variables in understanding health-related practices among 2SGBQ+ 
men. ${ }^{137-3945}$ Little is known about which demographics and socioecological factors associated with having a regular healthcare provider or a healthcare provider in the past 12 months. The present study is the first study in Manitoba that sought to address this gap in knowledge. We hypothesise that demographic and socioecological factors (discrimination in healthcare and perceived health practitioner's competence and knowledge of 2SGBQ+ mens' health issues) will be associated with healthcare access challenges (measured as having regular healthcare provider vs not, and having had a healthcare visit in the past 12 months vs not) for 2SGBQ+ men in Manitoba. The research question for this study is the following: for 2SGBQ+ men in Manitoba, what sociodemographics and socioecological factors are associated with: (1) having a regular healthcare provider and (2) having had a healthcare visit in the past 12 months.

\section{METHODOLOGY}

\section{Study design}

The data used in this paper were collected through a cross-sectional online survey method as part of the quantitative phase of the Manitoba Two-Spirit, Gay, Bisexual, and Queer Men's Health Study (www.manitobamensheal thstudy.com). The study was a community-based research study designed to examine the health and well-being of 2SGBQ+ men and their access to healthcare (including HIV care) in Manitoba. The study was conducted in collaboration with a community advisory committee (CAC) and the research team. The research team consisted of members connected to HIV/AIDS, sexual health and 2SLGBTQI+ community-based organisations (CBOs). These organisations worked hand in hand with our team throughout this research project. The CAC consisted of five $2 \mathrm{SGBQ}+$ men, representing diverse $2 \mathrm{SGBQ}+$ communities and assisted the research team with the development of the survey, recruitment and data analysis.

\section{Patient and public involvement}

Patients were not involved in this research. However, the study was conceptualised and developed while working closely with our community partners from the health and social sector and our CAC. Community members helped the research team with study implementation and advised on the recruitment and promotion of the research study.

\section{Recruitment, sampling and eligibility}

Participants $(n=368)$ for the online survey were recruited across Manitoba, using printed flyers (figure 1) at CBOs, word of mouth and through Facebook. CBOs also helped in recruiting $2 \mathrm{SGBQ}+$ men with diverse characteristics and from harder to reach populations, such as two-spirit and Indigenous $\mathrm{GBQ}+$ men, racialised $2 \mathrm{SGBQ}+$ men, young adult $2 \mathrm{SGBQ}+$ men, $2 \mathrm{SGBQ}+$ men living in rural settings and $2 \mathrm{SGBQ}+$ men living with HIV. Therefore, a large sample of 368 2SGBQ+ men for the online survey was needed as participants were selected based on these

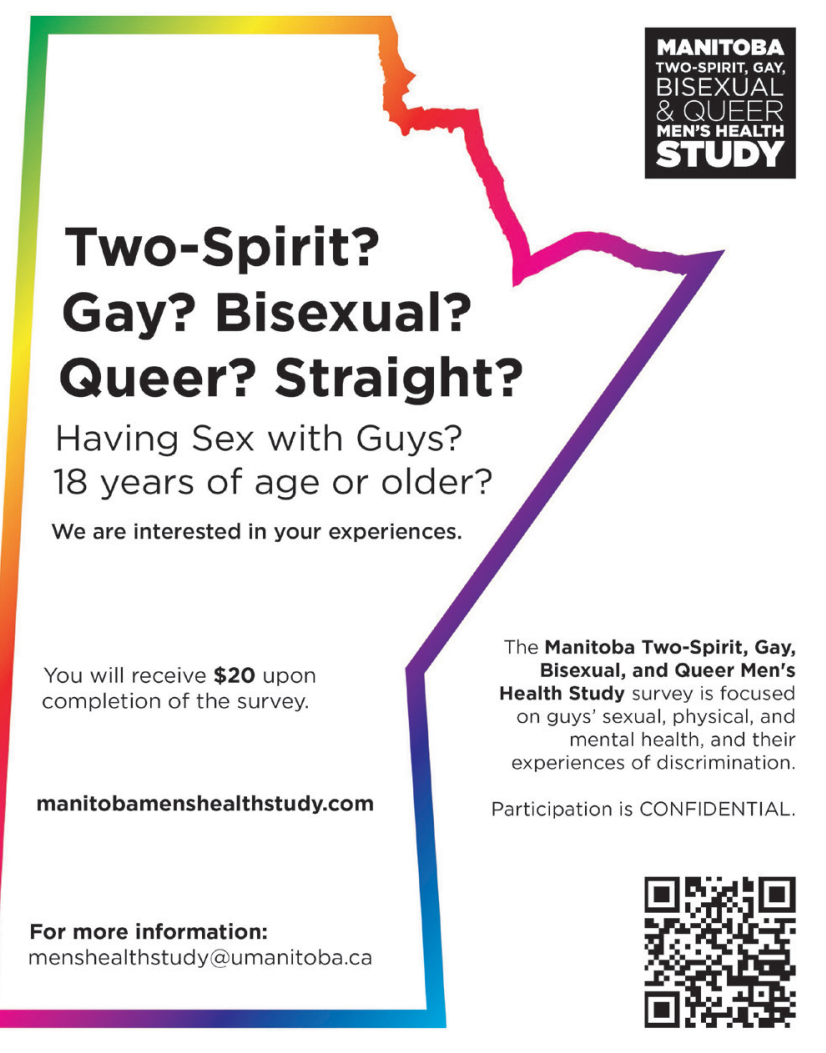

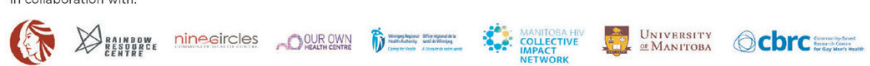

Figure 1 Recruitment flyer.

sociodemographics. Eligibility included: (1) identify as a man (cisgender or transgender), (2) report any sex with another man in the previous 12 months or identify as twospirit, gay, bisexual or queer, (3) be 18 years of age or older and (4) live or work in Manitoba. All participants were engaged in informed consent prior to beginning the study. All data were kept confidential. Survey participants were compensated \$C20.

\section{Measures}

The online survey included questions on demographics, sociostructural determinants of health, experiences of discrimination, health practitioner's competence and knowledge of $2 \mathrm{SGBQ}+$ men's health issues and access to healthcare in Manitoba.

\section{Demographics}

Sociodemographics measures included: (1) age; (2) race/ethnicity (black, African, Caribbean, East/Southeast Asian, First Nations, Métis, Latino, Latin American, white/Caucasian - Western European and white/ Caucasian - Eastern European); (3) sexual orientation identity (gay, bisexual, queer and other); (4) household income (\$0-\$29 999, \$30000-\$59 999, \$60 000-\$100 000, $\$ 100000+)$; (5) highest level of education completed (completed high school, bachelor's degree and graduate degree); and (6) geographic region where participants lived (large urban centre, medium city/town, small city/ 
town, smaller city/town, rural area close to city, rural and remote area, and on-reserve).

\section{Experiences of discrimination in healthcare}

To understand experiences of discrimination in healthcare, we asked participants: 'Over the past twoyears have you experienced discrimination in healthcare settings?' (response options included: 'yes', 'no'). Discrimination was operationalised in the survey as being treated badly or unfairly, denied equal treatment or services, verbally harassed or disrespected or physically assaulted or attacked.

\section{Perceived health practitioner's 2SGBQ+ competence and} knowledge

To measure respondents' perceptions of their healthcare provider's competence and knowledge of health issues affecting 2SGBQ+ men, we asked participants: 'How would you evaluate your doctor's (or other health practitioner's) competence and knowledge of issues affecting 2SGBQ+ men's health?'. Responses included: very good, good, fair, poor and very poor.

\section{Healthcare access variables (outcome variables)}

The following two outcome indicators of healthcare access were chosen in this analysis: (1) whether an individual has had a healthcare visit in the past 12 months, and (2) whether they had regular access to healthcare providers. These indicators are common measures in research ${ }^{38} 49$ used to assess 2SGBQ+ men's healthcare access. First, to assess if participants had visited healthcare providers in the past 12 months, we asked participants: 'In the past 12 months have you seen a healthcare provider/professional about your health?' (responses included: 'yes', 'no'). To establish if participants had a regular healthcare provider/family doctor, we asked: 'Do you have a regular family doctor or health practitioner?' ('yes', 'no').

\section{Data analyses}

All data analyses were conducted using SPSS V.27 (IBM Corp. 2020). First, descriptive analyses and tests were conducted. Descriptive analyses included means, SD for continuous variables (eg, age) as well as frequencies and proportions for categorical variables. Second, bivariate analyses were conducted using $\chi^{2}$ tests for categorical variables, as well as t-tests for continuous variables. After significant associations were identified in bivariate tests, multivariable analyses were conducted using binary logistic regression with reported ORs and 95\% CIs. A p value of less than $0.05(\mathrm{p}<0.05)$ was considered significant. The first logistic regression analysis was used to examine the relationship between having a regular healthcare provider versus not (outcome/dependent variable), socio demographics including age, ethnicity, education, household income, sexual orientation identity, geographic location (large urban centre, medium city/town, small city/town, smaller city/town, rural area close to city, rural and remote area, and on-reserve) and socioecological factors (discrimination, competence/knowledge). The second logistic regression analysis was used to examine the relationship between the healthcare visit in the past 12 months versus not (outcome/dependent variable), and sociodemographics and socioecological/structural factors as in the first logistic model. In the logistic regression model variables were entered using two blocks. In block one, the sociodemographic variables were entered. In block two, the theoretical variable of interest (such as experiences of healthcare discrimination and health practitioner's knowledge and competence) was entered.

\section{RESULTS}

\section{Participant characteristics and descriptive data}

Demographics (participant characteristics) and descriptive data $(n=368)$ on healthcare access are presented in table 1 . The mean age was 32.5 years $(\mathrm{SD}=8.4)$.

\section{Factors associated with having a regular healthcare provider/ family doctor}

In the bivariate analysis, there was a significant association between having a regular family doctor/healthcare provider and the geographic area where participants lived $\left(\chi^{2}=43.32, d f=6, p=0.001\right)$, such that participants who reported living in Winnipeg (large urban centre) were also more likely to report having a regular family doctor/ healthcare provider. No other demographic variables emerged as significant. The relationship between having a regular doctor/healthcare provider and experiences of discrimination was approaching significance $\left(\chi^{2}=9.25\right.$, $d f=4, \quad \mathrm{p}=0.055)$, where $2 \mathrm{SGBQ}+$ menwho reported discrimination also were more likely to report not having a regular provider. Finally, there was a significant association between having a regular family doctor/healthcare provider and healthcare providers' competence/knowledge of $2 \mathrm{SGBQ}+$ men's issues $\left(\chi^{2}=18.95, d f=4, \mathrm{p}=0.001\right)$, where participants who indicated healthcare providers' competence/knowledge of $2 \mathrm{SGBQ}+$ men's issues as 'very good' and 'good' were more likely to have access to regular healthcare providers/doctors.

\section{Factors associated with having a healthcare visit in the past 12 months}

In the bivariate analysis, there was a significant association between having a healthcare visit in the past 12 months and sexual orientation identity $\left(\chi^{2}=18.50, d f=3, \mathrm{p}=0.001\right)$, such that participants who self-identified as bisexual were more likely to have had a healthcare visit in the past 12 months. The relationship between having a healthcare visit in the past 12 months and the geographic area where participants lived also emerged as significant $\left(\chi^{2}=54.07\right.$, $d f=6, \mathrm{p}=0.001$ ), with people who lived in Brandon (medium sized city with a population under 50000 ) were less likely to have had a healthcare visit in the past 12 months compared with those $2 \mathrm{SGBQ}+$ men who lived in Winnipeg. No other demographic variables emerged as significant. Finally, a significant association was found between having a healthcare visit in the past 12 months 
Table 1 Sociodemographic characteristics and descriptive findings

\begin{tabular}{|c|c|c|}
\hline Characteristic & Frequency (n) & Percentage (\%) \\
\hline \multicolumn{3}{|l|}{ Ethnicity } \\
\hline $\begin{array}{l}\text { White - Western } \\
\text { European }\end{array}$ & 145 & 38.4 \\
\hline $\begin{array}{l}\text { White - Eastern } \\
\text { European }\end{array}$ & 68 & 18 \\
\hline $\begin{array}{l}\text { Black, African, } \\
\text { Caribbean }\end{array}$ & 41 & 10.8 \\
\hline First Nations & 40 & 10.6 \\
\hline Métis & 30 & 7.9 \\
\hline East/Southeast Asian & 29 & 7.7 \\
\hline Latino, Latin American & 25 & 6.6 \\
\hline \multicolumn{3}{|l|}{ Sexual orientation } \\
\hline Gay & 276 & 70.1 \\
\hline Bisexual & 68 & 17.3 \\
\hline Queer & 39 & 9.9 \\
\hline $\begin{array}{l}\text { Other (pansexual, } \\
\text { asexual) }\end{array}$ & 11 & 2.8 \\
\hline
\end{tabular}

Household income $(\$ C)$

\begin{tabular}{lrl}
$0-29999$ & 96 & 24.4 \\
$30000-59999$ & 77 & 19.6 \\
$60000-100000$ & 135 & 34.4 \\
100000 & 85 & 21.6 \\
\hline
\end{tabular}

\begin{tabular}{lrr}
$\begin{array}{l}\text { Highest education level } \\
\text { completed }\end{array}$ & & \\
\hline Completed high school & 99 & 24.9 \\
\hline Bachelor's degree & 271 & 68.3 \\
\hline Graduate degree & 27 & 6.8
\end{tabular}

\begin{tabular}{|c|c|c|}
\hline \multicolumn{3}{|c|}{ Regions where participants lived } \\
\hline $\begin{array}{l}\text { Large urban centre/ } \\
\text { Winnipeg (50 000+) }\end{array}$ & 249 & 61.5 \\
\hline $\begin{array}{l}\text { Medium city/town/ } \\
\text { Brandon ( } 30000-49000 \\
\text { people) }\end{array}$ & 54 & 13.3 \\
\hline $\begin{array}{l}\text { Small city/town (15 } \\
000-29 \text { 999) }\end{array}$ & 20 & 4.9 \\
\hline $\begin{array}{l}\text { Smaller city/town (1 } \\
000-14 \text { 999) }\end{array}$ & 34 & 8.4 \\
\hline $\begin{array}{l}\text { Rural area close to a } \\
\text { city }(<1000)\end{array}$ & 28 & 6.9 \\
\hline $\begin{array}{l}\text { Rural and remote area } \\
(<1000)\end{array}$ & 10 & 2.5 \\
\hline On-reserve & 10 & 2.5 \\
\hline \multicolumn{3}{|c|}{ Experienced discrimination in healthcare } \\
\hline Yes & 224 & 65.3 \\
\hline No & 119 & 34.7 \\
\hline
\end{tabular}

Doctor's/healthcare practitioner's competence with 2SGBQ+

\begin{tabular}{rrr} 
Very good $\quad 66$ & 18.2 \\
\hline & Continued
\end{tabular}

Table 1 Continued

\begin{tabular}{lcc}
\hline Characteristic & Frequency (n) & Percentage (\%) \\
\hline Good & 91 & 25.1 \\
Fair & 116 & 32 \\
Poor & 27 & 7.5 \\
Very poor & 12 & 3.3 \\
Don't know & 50 & 13.8 \\
Has regular family doctor/healthcare provider \\
Yes & 263 & 71.3 \\
No & 106 & 28.7
\end{tabular}

Seen healthcare provider in the past 12 months

\begin{tabular}{lrr} 
Yes & 293 & 79.1 \\
No & 77 & 20.8 \\
\hline
\end{tabular}

Total numbers vary due to missing data.

2SGBQ+, two-spirit, gay, bisexual, queer.

and perceptions of healthcare providers' competence/ knowledge of $2 \mathrm{SGBQ}+$ men's issues $\left(\chi^{2}=24.89, d f=4\right.$, $\mathrm{p}=0.001$ ), where participants who indicated healthcare providers' competence/knowledge of 2SGBQ+ men's issues as 'very good' and 'good' were more likely to have seen a healthcare provider in the past 12 months.

\section{Multivariate analyses}

The results of the logistic analysis revealed a significant logistic regression model for access to regular doctor/ healthcare provider for this sample of $2 \mathrm{SGBQ}+$ men in Manitoba $\left(\chi^{2}=57.24, \mathrm{p}<0.001\right)$. This model had a very good fit with the sample data $(-2 \log$ likelihood $=275.94$, Hosmer and Lemeshow $\chi^{2}$ test of goodness-of-fit, $\chi^{2}=9.08$, $\mathrm{p}>0.05$, Nagelkerke $R^{2}=0.26$ ). The model successfully predicted $79.4 \%$ of the cases. No interactions emerged as significant.

The results of the logistic analysis also revealed a significant logistic regression model for having a healthcare visit in the past 12 months for this sample of $2 \mathrm{SGBQ}+$ men in Manitoba $\left(\chi^{2}=88.24, \mathrm{p}<0.001\right)$. This model had a very good fit with the sample data $(-2 \log$ likelihood $=199.07$, Hosmer and Lemeshow $\chi^{2}$ test of goodness-of-fit, $\chi^{2}=6.80$, $\mathrm{p}>0.05$, Nagelkerke $\left.R^{2}=0.42\right)$. This model successfully predicted $86.9 \%$ of the cases. No interactions emerged as significant.

The results of the logistic regression analyses are presented in tables 2 and 3 .

\section{Factors associated with access to regular healthcare providers and healthcare visits in the past 12 months}

The results of the first logistic regression analyses revealed the significant association between the geographic area where participants lived, healthcare providers' competence/knowledge of 2SGBQ+ men's issues and access to regular doctor/healthcare provider (see table 2). Specifically, participants who lived in Brandon (a medium size town of 30000-49000 people in Manitoba) 
Table 2 Multiple logistic regression of sociodemographics, healthcare discrimination and healthcare providers' 2SGBQ+ competence on access to regular doctor/healthcare provider among 2SGBQ+ men in Manitoba $(n=368)$

\begin{tabular}{|c|c|c|c|c|c|}
\hline & B & SE & AOR & $95 \% \mathrm{Cl}$ & Wald statistic \\
\hline \multicolumn{6}{|l|}{ Sociodemographic variables } \\
\hline Age & 0.38 & 0.32 & 1.04 & 0.98 to 1.11 & 1.43 \\
\hline \multicolumn{6}{|l|}{ Race (ref=white/Western European) } \\
\hline White/Eastern European & 0.23 & 0.55 & 1.26 & 0.42 to 3.72 & 0.17 \\
\hline Black, African, Caribbean & 0.71 & 0.65 & 2.03 & 0.57 to 7.23 & 1.20 \\
\hline First Nations & 0.60 & 0.94 & 0.55 & 0.09 to 3.42 & 0.42 \\
\hline Métis & 0.08 & 0.84 & 1.09 & 0.21 to 5.57 & 0.01 \\
\hline East and Southeast Asian & 0.59 & 0.72 & 0.56 & 0.14 to 2.32 & 0.62 \\
\hline Latino, Latin American & 0.21 & 0.83 & 0.81 & 0.16 to 4.07 & 0.68 \\
\hline \multicolumn{6}{|l|}{ Household income $($ ref $=\$ C<30000)$} \\
\hline $30000-59999$ & -1.36 & 0.81 & 0.26 & 0.53 to 1.24 & 2.86 \\
\hline $60000-99999$ & -1.79 & 0.86 & 0.17 & 0.31 to 1.90 & 4.35 \\
\hline $100000+$ & -1.99 & 0.91 & 1.14 & 0.23 to 1.80 & 4.86 \\
\hline \multicolumn{6}{|l|}{ Education level (ref=completed high school) } \\
\hline Bachelor's degree & 0.53 & 0.60 & 1.70 & 0.52 to 5.50 & 0.78 \\
\hline Graduate degree & 1.70 & 1.06 & 5.46 & 0.68 to 43.50 & 2.57 \\
\hline \multicolumn{6}{|l|}{ Sexual orientation (ref=gay/homosexual) } \\
\hline Bisexual & 1.23 & 0.68 & 3.42 & 0.91 to 12.90 & 3.30 \\
\hline Queer & 0.59 & 0.78 & 1.81 & 0.40 to 8.37 & 0.58 \\
\hline Other (pansexual, asexual) & 0.58 & 1.16 & 1.80 & 0.19 to 17.32 & 0.26 \\
\hline \multicolumn{6}{|l|}{ Geographic area (ref=large urban centre/Winnipeg) } \\
\hline Medium city/town (30000-49 000 people) & -2.52 & 0.51 & $0.08^{\star *}$ & 0.03 to 0.22 & 23.6 \\
\hline Small city/town (15 000-29 999) & -1.61 & 0.82 & $0.20^{*}$ & 0.04 to 0.98 & 3.92 \\
\hline Smaller city/town (1000-14-999) & -1.34 & 0.57 & $0.26^{*}$ & 0.09 to 0.81 & 5.45 \\
\hline Rural area close to a city $(<1000)$ & 0.46 & 0.85 & 0.59 & 0.30 to 8.38 & 0.30 \\
\hline Rural and remote area not close to a city $(<1000)$ & 0.32 & 0.54 & 0.46 & 0.33 to 9.56 & 0.10 \\
\hline On-reserve & -0.65 & 1.08 & 0.52 & 0.06 to 4.36 & 0.36 \\
\hline \multicolumn{6}{|l|}{ Sociostructural variables } \\
\hline Discrimination in healthcare & -0.40 & 0.49 & 0.67 & 0.28 to 1.74 & 0.68 \\
\hline
\end{tabular}

Healthcare providers' 2 SGBQ+ competence

(ref=very good)

\begin{tabular}{llllll} 
Good & -0.90 & 0.70 & 0.41 & 0.10 to 1.59 & 1.67 \\
Fair/somewhat OK & -0.58 & 0.69 & 0.56 & 0.14 to 2.20 & 0.69 \\
Poor & -1.68 & 0.80 & $\mathbf{0 . 1 9 ^ { * }}$ & 0.03 to 0.90 & 4.40 \\
Very poor & -3.57 & 1.11 & $\mathbf{0 . 0 3}$ & 0.01 to 0.25 & 10.3 \\
\hline
\end{tabular}

${ }^{*} \mathrm{P}<0.05,{ }^{* *} \mathrm{P}<0.001$.

Bold text indicates statistical significance.

AOR, adjusted OR; Ref., reference group; 2SGBQ+, two-spirit, gay, bisexual, queer.

compared with Winnipeg were significantly less likely to have access to a regular doctor/healthcare provider (AOR $=0.08, \mathrm{SE}=0.52,95 \%$ CI 0.03 to $0.22, \mathrm{p}=0.001)$. In addition, compared with those who lived in Winnipeg, participants who lived in small cities with 15000-29999 people (AOR=0.20, $\mathrm{SE}=0.82,95 \%$ CI 0.04 to $0.98, \mathrm{p}=0.05$ ) and smaller towns with $1000-14999$ people $(\mathrm{AOR}=0.26$, $\mathrm{SE}=0.57,95 \%$ CI 0.08 to $0.81, \mathrm{p}=0.05$ ) were less likely to have access to regular healthcare providers. No other demographics emerged as significant. Compared with 2SGBQ + men who indicated their healthcare providers' competence/knowledge of 2SGBQ+ men's issues as 'very good', those participants who indicated their healthcare providers' competence/knowledge as 'poor' ( $\mathrm{AOR}=0.19$, $\mathrm{SE}=0.80,95 \%$ CI 0.04 to $0.90, \mathrm{p}=0.05$ ) and 'very poor' (AOR=0.03, $\mathrm{SE}=0.11,95 \%$ CI 0.03 to $0.25, \mathrm{p}=0.001)$ were 
Table 3 Multiple logistic regression of sociodemographics, healthcare discrimination and healthcare providers' 2SGBQ+ competence on healthcare visits in the past 12 months among 2SGBQ+ men in Manitoba $(n=368)$

\begin{tabular}{|c|c|c|c|c|c|}
\hline & B & SE & AOR & $95 \% \mathrm{Cl}$ & Wald statistic \\
\hline \multicolumn{6}{|l|}{ Sociodemographic variables } \\
\hline Age & -0.01 & 0.03 & 0.99 & 0.93 to 1.07 & 0.02 \\
\hline \multicolumn{6}{|l|}{ Race (ref=white/Western European) } \\
\hline White/Eastern European & 0.83 & 0.67 & 2.29 & 0.62 to 8.45 & 1.54 \\
\hline Black, African, Caribbean & 1.13 & 0.74 & 3.09 & 0.72 to 13.20 & 2.31 \\
\hline First Nations & 1.91 & 1.39 & 6.76 & 0.44 to 103.54 & 1.88 \\
\hline Métis & 2.20 & 1.22 & 8.99 & 0.82 to 98.33 & 3.23 \\
\hline East and Southeast Asian & -0.87 & 0.72 & 0.42 & 0.10 to 1.71 & 1.47 \\
\hline Latino, Latin American & -0.75 & 0.84 & 0.47 & 0.09 to 2.45 & 0.80 \\
\hline \multicolumn{6}{|l|}{ Household income $(r e f=\$ C<30000)$} \\
\hline $30000-<59999$ & 2.12 & 1.03 & 8.30 & 1.10 to 62.86 & 4.20 \\
\hline $60000-99999$ & -0.10 & 0.71 & 0.90 & 0.23 to 3.61 & 0.02 \\
\hline $100000+$ & 0.19 & 0.60 & 1.20 & 0.37 to 3.90 & 0.09 \\
\hline \multicolumn{6}{|l|}{ Education level (ref=completed high school) } \\
\hline Bachelor's degree & 0.82 & 0.70 & 2.27 & 0.58 to 8.90 & 1.39 \\
\hline Graduate degree & 0.71 & 1.02 & 2.03 & 0.28 to 14.82 & 0.49 \\
\hline \multicolumn{6}{|l|}{ Sexual orientation (ref=gay/homosexual) } \\
\hline Bisexual & 2.53 & 0.97 & $12.57^{\star}$ & 1.88 to 83.97 & 6.83 \\
\hline Queer & 0.46 & 0.93 & 1.58 & 0.25 to 9.91 & 0.25 \\
\hline Other (pansexual, asexual) & 1.27 & 1.47 & 0.28 & 0.02 to 5.06 & 1.48 \\
\hline \multicolumn{6}{|l|}{ Geographic area (ref=large urban centre/Winnipeg) } \\
\hline Medium city/town (30000-49 000 people) & -2.99 & 0.62 & $0.05^{\star \star}$ & 0.01 to 0.17 & 23.33 \\
\hline Small city/town (15 000-29 999) & -1.90 & 0.99 & 0.15 & 0.02 to 1.04 & 3.68 \\
\hline Smaller city/town (1000-14 999) & -1.41 & 0.66 & $0.24^{*}$ & 0.07 to 0.90 & 4.48 \\
\hline Rural area close to a city $(<1000)$ & -1.32 & 0.69 & 0.26 & 0.70 to 1.05 & 3.59 \\
\hline Rural and remote area not close to a city $(<1000)$ & -1.88 & 0.62 & 0.58 & 0.33 to 9.56 & 1.10 \\
\hline On-reserve & -1.25 & 1.44 & 0.28 & 0.02 to 4.88 & 0.74 \\
\hline \multicolumn{6}{|l|}{ Sociostructural variables } \\
\hline Discrimination in healthcare & -0.02 & 0.59 & 0.98 & 0.31 to 3.10 & 0.01 \\
\hline \multicolumn{6}{|l|}{$\begin{array}{l}\text { Healthcare providers' } 2 \text { SGBQ+ competence } \\
\text { (ref=very good) }\end{array}$} \\
\hline Good & -0.43 & 0.80 & 0.65 & 0.13 to 3.10 & 0.29 \\
\hline Fair/somewhat OK & -0.73 & 0.78 & 0.48 & 0.10 to 2.25 & 0.86 \\
\hline Poor & -1.49 & 0.92 & 0.24 & 0.04 to 1.41 & 2.50 \\
\hline Very poor & -1.19 & 1.17 & 0.31 & 0.03 to 3.07 & 1.02 \\
\hline
\end{tabular}

${ }^{*} \mathrm{P}<0.05,{ }^{* *} \mathrm{p}<0.001$.

Bold text indicates statistical significance.

AOR, adjusted OR; Ref., reference group; 2SGBQ+, two-spirit, gay, bisexual, queer.

less likely to have access to regular doctor/healthcare provider.

The results of the second logistic regression analyses revealed the significant association between the geographic area where participants lived, sexual orientation identity and having a healthcare visit in the past 12 months (see table 3). Specifically, compared with 2SGBQ+ men who lived in Winnipeg, participants who lived in Brandon, a medium size town of 30000-50000 people (AOR $=0.05, \mathrm{SE}=0.62,95 \%$ CI 0.02 to $0.17, \mathrm{p}=0.001$ ) and smaller towns of $1000-14999$ people $(\mathrm{AOR}=0.25, \mathrm{SE}=0.66$, $95 \%$ CI 0.67 to $0.90, \mathrm{p}=0.05$ ) were significantly less likely to have access to have had a healthcare visit in the past 12 months. Finally, participants who self-identified as 
bisexual were more likely to report having had a healthcare visit in the past 12 months compared with participants who self-identified as gay $(\mathrm{AOR}=12.57, \mathrm{SE}=0.97$, $95 \%$ CI 1.88 83.97, $\mathrm{p}=0.005$ ).

\section{DISCUSSION}

This study explored factors associated with healthcare access among 2SGBQ+ men in Manitoba, an understudied population in Canada. Notably, we found that $65.3 \%$ of the sample reported experiencing discrimination in healthcare settings in Manitoba. Furthermore, 28.7\% of the sample reported not having a regular healthcare provider, and $20.8 \%$ have not seen a healthcare provider about their health in the past 12 months.

This study showed an association between sociodemographic, socioecological factors and indicators of healthcare access among a sample of 2SGBQ+ men in Manitoba. First, this study found a significant association between the geographic area where participants lived, healthcare providers' competence and knowledge of $2 \mathrm{SGBQ}+$ men's issues and access to a regular doctor/healthcare provider. Compared with participants who lived in Winnipeg, those that lived in Brandon and smaller towns in Manitoba were significantly less likely to report having a regular doctor/ healthcare provider. Furthermore, compared with 2SGBQ+ men who indicated their healthcare providers' competence/knowledge of 2SGBQ+ men's issues as 'very good', those participants who indicated their healthcare providers' competence/knowledge as 'poor' and 'very poor' were less likely to report having a regular doctor/ healthcare provider.

Second, our study revealed the significant association between the geographic area where participants lived, sexual orientation identity and having a healthcare visit in the past 12 months. Compared with $2 \mathrm{SGBQ}+$ men who lived in Winnipeg, participants who lived in Brandon and other smaller cities/towns were significantly less likely to have had a healthcare visit in the past 12 months. Furthermore, participants who self-identified as bisexual were more likely to report having had a healthcare visit in the past 12 months compared with gay-identified participants.

This study is also not without limitations. First, this study relies on self-reported data and are subject to social desirability, recall and information bias. Second, the data relied on cross-section survey design, which presents issues with establishing directionality of findings. Furthermore, it is important to point out that there are other factors (oppression and social locations) not measured here that may be critical in understanding the relationship among the study variables. For instance, structural and interpersonal discrimination in the form of stigma or racism among $2 \mathrm{SGBQ}+$ men may result in socioeconomic practices that limit access to healthcare with potential effects on health and well-being. ${ }^{10}$ Future studies also need to take this into account other factors (mental health and disabilities) that may play a role in healthcare access. Nevertheless, the findings from this research are consistent with other literature on this topic. Similar to other research, ${ }^{51}$ our findings support a multilevel approach, contribute to a socioecological understanding of healthcare barriers and underscore the importance of attending to sociodemographic and socioecological/ structural factors.

The project employed socioecological framework to examine the effects of sociodemographic, interpersonal and structural factors and the ways in which these affect the healthcare access of cis and trans 2SGBQ+ men, emphasising the multifaceted nature of person-environment influences in the socioecological framework. This study's findings point to sociodemographic (geographic area where participants lived and sexual orientation identity) and socioecological/structural factors (healthcare providers competence and knowledge of $2 \mathrm{SGBQ}+$ men's health issues) that are associated with indicators of healthcare access, therefore contributing to a socioecological understanding of healthcare barriers and facilitators among $2 \mathrm{SGBQ}+$ men. Furthermore, the socioecological model used in this study provided a structure for interpreting the findings that may also help guide interventions to address healthcare among this population.

With regards to geographic location and access to healthcare, our findings indicated that healthcare access is an issue for participants who lived in Brandon and other smaller cities and towns in Manitoba. This finding is consistent with previous research, ${ }^{4656}$ which suggests that stigma and social isolation in smaller, remote and rural areas may be at play when it comes to individuals' access to healthcare. Though no data exist thus far on 2SGBQ+ men who live in Brandon or smaller towns in Manitoba, at first glance, rural areas appear less hospitable to 2SGBQ+ men. This is the first study in Manitoba to point in this direction. Given the geographic isolation of northern and remote communities in Manitoba, 2SGBQ+ men may also face a complex set of barriers to healthcare that includes geography, lack of trust, lack of transportation, as well as lower number of healthcare providers in these areas. All of these factors may explain our findings that indicate that healthcare access is an issue for participants who lived in Brandon and other smaller cities and towns in Manitoba. Future research needs to elucidate the impacts of sexuality and/or gender minority stigma on $2 \mathrm{SGBQ}+$ men's access in smaller and remote towns in Manitoba. It is important to reduce the gap between the healthcare access of rural and urban 2SGBQ+ men. The availability of services for sexual and gender minority people in Brandon and other smaller communities (where 2SGBQ+ men may feel more stigmatised) is also an issue that needs to be brought to the attention of provincial and territorial healthcare. Furthermore, creation of antidiscriminatory health policies at the governmental and institutional level to facilitate the development of equitable and accessible health services for $2 \mathrm{SGBQ}+$ men in Manitoba is urgently needed.

Our findings are also consistent with the line of research that shows that healthcare providers' competence and 
knowledge of issues affecting 2SGBQ+ men are closely associated with healthcare access and utilisation. ${ }^{57-59}$ These findings underscore the importance of addressing healthcare providers lack of knowledge or cultural competence in healthcare settings as it may act as a barrier to healthcare. Various elements can improve or facilitate 2SGBQ+ men's healthcare, including: the creation of a safe, 2SGBQ-affirming space free from violence and discrimination that $2 \mathrm{SGBQ}+$ men frequently face; the development of trust in relationships and respect of privacy during service delivery; the use of outreach services to $2 \mathrm{SGBQ}+$ men's communities; and the provision of culturally competent care and referrals. ${ }^{575}$ Mayer and colleagues ${ }^{59}$ work on integrated comprehensive care for sexual and gender minority men emphasises culturally competent care based in human rights principles and stresses the importance of gathering the appropriate information to be able to provide care and make referrals and developing a strong rapport with $2 \mathrm{SGBQ}+$ men in order to maintain a regular access to healthcare. Given the findings on the differences between gay and bisexual men, health and social care service providers may also require training and opportunities to increase information and skills to prepare them to adequately support gayidentified men in healthcare contexts.

The findings from this study also paint a picture where bisexual men were more likely to report having had a healthcare visit in the past 12 months compared with participants who self-identified as gay. Although some scholarship suggested that gay and bisexual men do not differ significantly from one another in terms of health practices, ${ }^{60}$ other research pointed out differences between gay and bisexual-identified men. ${ }^{61}$ This finding adds to the knowledge base on the differences between bisexual and gay-identified men. ${ }^{62}$ While some research shows that bisexual men experience more marginalisation in healthcare compared with gay men, ${ }^{62}$ our study presented a different picture. Given that health services are typically culturally insensitive towards the needs of gay men (coupled with concerns about homophobia), this may discourage some sexual minority people from identifying as gay (as opposed to bisexual) in order to avoid the associated stigma. It is therefore essential for future research to explore how the social context toward gay and bisexual men (in mainstream communities and society at large) increases gay men's invisibility and further decreases health access and uptake for gay men.

Our results are different from those by McKirnan and colleagues ${ }^{38}$ in that their study (conducted in USA) found that lower incomes were associated with less healthcare access among men who have sex with men. Our findings did not show an association between lower incomes and access to healthcare among this sample of 2SGBQ+ men. One explanation for this discrepancy may relate to the fact that our research was conducted in Canada, which has free universal access to healthcare.

Importantly, our findings did not show an association between discrimination in healthcare and access to healthcare among a sample of $2 \mathrm{SGBQ}+$ men in Manitoba, after controlling for sociodemographics. This area of inquiry highlights important results by suggesting that a relationship between discrimination and its effects on healthcare access among $2 \mathrm{SGBQ}+$ menmay be more complex than previously theorised, particularly in Western contexts such as Canada. Future research needs to further explore the relationship between these variables. Furthermore, given that discrimination among $2 \mathrm{SGBQ}+$ men in healthcare may exist in multiple forms and stem from multiple sources, our study was limited in the way discrimination was measured. For our measurement, we asked participants if they experienced discrimination in healthcare settings over the past 2 years. Instead, there may be a need to accurately and comprehensively assess the multidimensional facets of discrimination among 2SGBQ+ men. This would require paying greater attention to: (1) capturing the dimensions of discriminatory experiences in healthcare, including the severity, chronicity and duration of these experiences, (2) the need to expand assessment to capture discrimination affecting $2 \mathrm{SGBQ}+$ men in multiple domains (eg, race and ethnicity, sexual identity, gender identity or expression and socioeconomic status) and (3) the need to understand the intersectional nature of discrimination, stigma and social exclusion of 2SGBQ+ men in healthcare contexts.

The demographic and socioecological factors identified in this study may influence $2 \mathrm{SGBQ}+$ men's current and future engagement with healthcare. Strategies that attend to these factors can help mitigate negative experiences among $2 \mathrm{SGBQ}+$ men in healthcare settings, ensure access to services and create affirming healthcare environments that support $2 \mathrm{SGBQ}+$ men in maintaining their health. There must also be recognition of the unique and specific health and well-being issues affecting 2SGBQ+ men in Manitoba. This recognition should be substantiated with evidence and followed by implementation of funding, programming and services, in which 2SGBQ+ men in Manitoba can be represented in health policy. Prioritising 2SGBQ+ men in order to address their health and well-being concerns, the healthcare and social service systems in Manitoba must reshape how they provide service delivery. Broader society needs to become knowledgeable and sensitised to these issues through education campaigns in order to improve access and combat homophobia, heterosexism, stigma and other forms of discrimination directed at $2 \mathrm{SGBQ}+$ men in Manitoba.

\section{Author affiliations}

${ }^{1}$ Faculty of Social Work, University of Manitoba, Winnipeg, Manitoba, Canada ${ }^{2}$ Department of Community Health Sciences, Rady Faculty of Health Sciences University of Manitoba, Winnipeg, Manitoba, Canada

${ }^{3}$ Two-Spirited People of Manitoba, Winnipeg, Manitoba, Canada

${ }^{4}$ Faculty of Education, University of Manitoba, Winnipeg, Manitoba, Canada

${ }^{5}$ Nine Circles Community Health Centre, Winnipeg, Manitoba, Canada

${ }^{6}$ Manitoba HIV-STBBI Collective Impact Network, Winnipeg, Manitoba, Canada ${ }^{7}$ Department of Internal Medicine, Rady Faculty of Health Sciences University of Manitoba, Winnipeg, Manitoba, Canada

${ }^{8}$ Department of Occupational Therapy, Rady Faculty of Health Sciences University of Manitoba, Winnipeg, Manitoba, Canada 
${ }^{9}$ Winnipeg Regional Health Authority, Winnipeg, Manitoba, Canada

${ }^{10}$ Our Own Health Centre, Winnipeg, Manitoba, Canada

${ }^{11}$ Community-Based Research Centre, Victoria, British Columbia, Canada

${ }^{12}$ School of Public Health and Social Policy, University of Victoria, Victoria, British Columbia, Canada

${ }^{13}$ Factor-Inwentash Faculty of Social Work, University of Toronto, Toronto, Ontario, Canada

Twitter Michael Payne @mikejamespayne, Nathan J Lachowsky @NJLachowsky and David J Brennan @DrDavidJBrennan

Contributors RS was responsible for the overall design of the study. All authors participated in discussions about the data source and planning of the analyses, and critically revised successive versions of the paper. RS drafted the first version of the manuscript. RS, JS, AM, SA, SM and CC were involved in the initial data analysis. RL, MP, LR, LL, GR, PM, BM, NJL, DJB and UNS provided input into the data analysis and interpretation. All coauthors were involved in the drafting various components of the manuscript. The study was conceptualised and developed while working closely with our community partners from the health and social sector and our community advisory committee. RS is responsible for the overall content as the guarantor.

Funding This work was supported by the Canadian Institutes of Health Research Catalyst Grant (\#162929), the Manitoba Medical Service Foundation operating grant (\#8-2019-12) and the Winnipeg Foundation operating grant (\#321813).

\section{Competing interests None declared.}

Patient and public involvement Patients and/or the public were not involved in the design, or conduct, or reporting, or dissemination plans of this research.

\section{Patient consent for publication Not applicable.}

Ethics approval This study involves human participants and was approved by University of Manitoba Research Ethics Board Protocol P2019027 HS22318. Participants gave informed consent to participate in the study before taking part.

Provenance and peer review Not commissioned; externally peer reviewed.

Data availability statement Data are available on reasonable request.

Open access This is an open access article distributed in accordance with the Creative Commons Attribution Non Commercial (CC BY-NC 4.0) license, which permits others to distribute, remix, adapt, build upon this work non-commercially, and license their derivative works on different terms, provided the original work is properly cited, appropriate credit is given, any changes made indicated, and the use is non-commercial. See: http://creativecommons.org/licenses/by-nc/4.0/.

\section{ORCID iDs}

Rusty Souleymanov http://orcid.org/0000-0002-2619-2704

Jared Star http://orcid.org/0000-0003-2875-515X

Nathan J Lachowsky http://orcid.org/0000-0002-6336-8780

David J Brennan http://orcid.org/0000-0002-7663-9869

\section{REFERENCES}

1 Michael T. Health care use among gay, lesbian and bisexual Canadians 2008

2 Travis SH, Olivier F, Dionne G. Suicide and HIV as leading causes of death among gay and bisexual men: a comparison of estimated mortality and published research 2015.

3 Ross LE, Bauer GR, MacLeod MA, et al. Mental health and substance use among bisexual youth and Non-Youth in Ontario, Canada 2014.

4 Public Health Agency of Canada. Summary: Estimates of HIV incidence, prevalence and Canada's progress on meeting the 9090-90 HIV targets, 2016. Surveillance and Epidemiology Division. Professional Guidelines and Public Health Practice Division. Centre for Communicable Disease and Infection Control. Public Health Agency of Canada, 2018.

5 Manitoba HIV Program. Manitoba HIV program report: 2016. Nine Circles Community Health Centre, 2017.

6 Yu D, Hatala AR, Reimer J. 'I'm more aware of my HIV risk than anything else': syndemics of syphilis and HIV among gay men in Winnipeg. Culture, Health \& Sexuality 2017:1-13.

7 Winnipeg Regional Health Authority (WRHA). Outbreak of infectious syphilis in the Winnipeg health region. Winnipeg: Winnipeg Health Region Press, 2014.
8 Public Health Agency of Canada. Reducing the health impact of sexually transmitted and blood-borne infections in Canada by 2030: a pan-Canadian STBBI framework for action. Centre for Communicable Diseases and Infection Control, Infectious Disease and Prevention and Control branch, Public Health Agency of Canada, 2018.

9 Brennan DJ, Souleymanov R, George C, et al. Masculinity, muscularity, and HIV sexual risk among gay and bisexual men of color. Psychol Men Masc 2015;16:393-403.

10 Souleymanov R, Brennan DJ, George C, et al. Experiences of racism, sexual objectification and alcohol use among gay and bisexual men of colour. Ethn Health 2020;25:525-41.

11 Keogh P, Henderson L, Dodds C. Ethnic minority gay men: redefining community, restoring identity, 2004. Available: https://www. researchgate.net/profile/Peter_Keogh/ publication/237281085_ Ethnic_Minority_Gay_Men_Redefining_Community_Restoring_ Identity/links/00b7d527be6e40eb27000000.pdf

12 Wolitski RJ, Stall R, Valdiserri RO, eds. Unequal opportunity: health disparities affecting gay and bisexual men in the United States. Oxford. USA: University Press, 2008.

13 Souleymanov R. Social workers at the frontiers of technology: Onlinebased HIV prevention and care for men who have sex with men. Geneva, Switzerland: International Association of Schools of Social Work \& Joint United Nations Program on HIV/AIDS, 2017. http:// www.unaids.org/en/resources/documents/2017/global-social-workresponds-to-HIV

14 Sandfort TGM, de Graaf R, Bijl RV. Same-sex sexuality and quality of life: findings from the Netherlands mental health survey and incidence study. Arch Sex Behav 2003;32:15-22.

15 Feldman MB, Meyer $\mathrm{IH}$. Childhood abuse and eating disorders in gay and bisexual men. Int J Eat Disord 2007;40:418-23.

16 Hart TA, Rotondi NK, Souleymanov R, et al. Psychometric properties of the social appearance anxiety scale among Canadian gay and bisexual men of color. Psychol Sex Orientat Gend Divers 2015;2:470-81.

17 Brennan DJ, Ross LE, Dobinson C, et al. Men's sexual orientation and health in Canada. Can J Public Health 2010;101:255-8.

18 de Graaf R, Sandfort TGM, ten Have M. Suicidality and sexual orientation: differences between men and women in a general population-based sample from the Netherlands. Arch Sex Behav 2006;35:253-62.

19 Gilman SE, Cochran SD, Mays VM, et al. Risk of psychiatric disorders among individuals reporting same-sex sexual partners in the National comorbidity survey. Am J Public Health 2001;91:933.

20 Sandfort TGM, Bakker F, Schellevis FG, et al. Sexual orientation and mental and physical health status: findings from a Dutch population survey. Am J Public Health 2006;96:1119-25.

21 Brennan DJ, Hellerstedt WL, Ross MW, et al. History of childhood sexual abuse and HIV risk behaviors in homosexual and bisexual men. Am J Public Health 2007;97:1107-12.

22 Newman PA, Rhodes F, Weiss RE. Correlates of sex trading among drug-using men who have sex with men. Am J Public Health 2004;94:1998-2003.

23 Adam BD, Hart TA, Mohr J, et al. HIV-related syndemic pathways and risk subjectivities among gay and bisexual men: a qualitative investigation. Cult Health Sex 2017;19:1254-67.

24 Hart TA, Noor SW, Adam BD, et al. Number of psychosocial strengths predicts reduced HIV sexual risk behaviors above and beyond syndemic problems among gay and bisexual men. AIDS Behav 2017;21:3035-46.

25 Stall R, Mills TC, Williamson J, et al. Association of co-occurring psychosocial health problems and increased vulnerability to HIV/ AIDS among urban men who have sex with men. Am J Public Health 2003;93:939-42.

26 Dyer TP, Shoptaw S, Guadamuz TE, et al. Application of syndemic theory to black men who have sex with men in the Multicenter AIDS cohort study. J Urban Health 2012;89:697-708.

27 Singer M, Clair S. Syndemics and public health: reconceptualizing disease in bio-social context. Med Anthropol Q 2003;17:423-41.

28 Singer MC, Erickson PI, Badiane L, et al. Syndemics, sex and the city: understanding sexually transmitted diseases in social and cultural context. Soc Sci Med 2006;63:2010-21.

29 Lee R. Health care problems of lesbian, gay, bisexual, and transgender patients. West J Med 2000;172:403-8.

30 Mayer KH, Bradford JB, Makadon HJ, et al. Sexual and gender minority health: what we know and what needs to be done. Am J Public Health 2008;98:989-95.

31 Institute of Medicine. The health of Lesbian, gay, bisexual, and transgender people: building a foundation for better Understand-ing. Washinton, DC: The National Academies Press, 2011. 
32 Sanchez NF, Rabatin J, Sanchez JP, et al. Medical students' ability to care for lesbian, gay, bisexual, and transgendered patients. Fam Med 2006;38:21-7.

33 Bergeron S, Senn CY. Health care utilization in a sample of Canadian lesbian women: predictors of risk and resilience. Women Health 2003;37:19-35.

34 Jackson NC, Johnson MJ, Roberts R. The potential impact of discrimination fears of older gays, lesbians, bisexuals and transgender individuals living in small- to moderate-sized cities on long-term health care. J Homosex 2008;54:325-39.

35 Safren SA, Reisner SL, Herrick A, et al. Mental health and HIV risk in men who have sex with men. J Acquir Immune Defic Syndr 2010;55(Suppl 2):S74-7.

36 Halkitis PN, Perez-Figueroa RE, Carreiro T, et al. Psychosocial burdens negatively impact HIV antiretroviral adherence in gay, bisexual, and other men who have sex with men aged 50 and older. AIDS Care 2014;26:1426-34.

37 Gee R. Primary care health issues among men who have sex with men. J Am Acad Nurse Pract 2006;18:144-53.

38 McKirnan DJ, Du Bois SN, Alvy LM, et al. Health care access and health behaviors among men who have sex with men: the cost of health disparities. Health Educ Behav 2013;40:32-41.

39 Tjepkema M. Health care use among gay, lesbian and bisexual Canadians. Health Rep 2008;19:53-64.

40 Müller A. Health for all? sexual orientation, gender identity, and the implementation of the right to access to health care in South Africa. Health Hum Rights 2016;18:195.

41 Statistics Canada. Canadian Community Health Survey

(CCHS) - Detailed information for 2005 (Cycle 3.1)

Available: http://www.statcan.gc.ca/cgi-bin/imdb/ p2SV. pl?Function=getSurvey\&Survld $=3226 \&$ SurvV er $=0 \&$ Instald=15282\&InstaV er=3\&SDDS=3226\&la $\mathrm{ng}=\mathrm{en} \& \mathrm{db}=\mathrm{imdb} \& \mathrm{adm}=8 \& \mathrm{dis}=2$ [Accessed 15 Jun 2010].

42 Statistics Canada Canadian Community Health Survey (CCHS). Detailed information for 2007 (cycle 4.1). Available: http://www.statcan.gc.ca/cgi-bin/imdb/ p2SV. pl?Function=getSurvey\&Survld $=3226 \&$ SurvV er $=1 \&$ Instald $=15282 \&$ InstaV er $=4 \& S D D S=3226 \& l a$ $\mathrm{ng}=\mathrm{en} \& \mathrm{db}=\mathrm{imdb} \& \mathrm{adm}=8 \& \mathrm{dis}=2$

43 Petroll AE, Mosack KE. Physician awareness of sexual orientation and preventive health recommendations to men who have sex with men. Sex Transm Dis 2011;38:63-7.

$44 \mathrm{Ng} \mathrm{BE}$, Moore D, Michelow W, et al. Relationship between disclosure of same-sex sexual activity to providers, HIV diagnosis and sexual health services for men who have sex with men (MSM) in Vancouver, Canada. Can J Public Health 2014;105:e186-91.

45 Shari B, Bill R, Yves J. Reclaiming Space-Regaining health. Journal of Gay \& Lesbian Social Services 2002
46 Swank E, Fahs B, Frost DM. Region, social identities, and disclosure practices as predictors of Heterosexist discrimination against sexual minorities in the United States. Sociol Inq 2013;83:238-58.

47 Kates J, Ranji U, Beamesderfer A. 'Health and Access to Care and Coverage for Lesbian, Gay, Bisexual, and Transgender Individuals in the US.', The Henry J. Kaiser Family Foundation, 2015.

48 Blondeel K, Say L, Chou D, et al. Evidence and knowledge gaps on the disease burden in sexual and gender minorities: a review of systematic reviews. Int J Equity Health 2016;15:16.

49 Alvy LM, McKirnan D, Du Bois SN, et al. Health care disparities and behavioral health among men who have sex with men. Journal of Gay Lesbian Social Services 2011;23:507-22.

50 Austin A, Herrick H, Proescholdbell S. Adverse childhood experiences related to poor adult health among lesbian, gay, and bisexual individuals. Am J Public Health 2016;106:314-20.

51 Lacombe-Duncan A, Logie CH, Li J, et al. Social-ecological factors associated with having a regular healthcare provider among lesbian, gay, bisexual and transgender persons in Jamaica. Glob Public Health 2021:1-14.

52 Shields SA. Gender: an intersectionality perspective. Sex Roles 2008;59:301-11.

53 Bronfenbrenner U. Ecological models of human development. International encyclopedia of education 1994;3:37-43.

54 Siporin M. Ecological systems theory in social work. J Sociol Soc Welfare 1980;7:507-32.

55 Baral S, Logie CH, Grosso A, et al. Modified social ecological model: a tool to guide the assessment of the risks and risk contexts of HIV epidemics. BMC Public Health 2013;13:482.

56 Whitehead J, Shaver J, Stephenson R. Outness, stigma, and primary health care utilization among rural LGBT populations. PLoS One 2016;11:e0146139.

57 Brotman S, Ryan B, Jalbert Y, et al. The impact of coming out on health and health care access: the experiences of gay, lesbian, bisexual and two-spirit people. J Health Soc Policy 2002;15:1-29.

58 Dodge B, Schnarrs PW, Goncalves G, et al. The significance of privacy and trust in providing health-related services to behaviorally bisexual men in the United States. AIDS Educ Prev 2012;24:242-56.

59 Mayer KH, Bekker L-G, Stall R, et al. Comprehensive clinical care for men who have sex with men: an integrated approach. Lancet 2012;380:378-87.

60 Klein H. Differences in HIV risk practices sought by self-identified gay and bisexual men who use Internet websites to identify potential sexual partners. J Bisex 2009;9:125-40.

61 Phillips G, Kalmin MM, Turner B, et al. Condom and substance use at last sex: differences between MSMO and MSWO high school youth. Int J Environ Res Public Health 2018;15:995.

62 Souleymanov R, Fantus S, Lachowsky N, et al. How bisexualidentified men use the Internet to seek sex with other men in Ontario: factors associated with HIV/STI testing and condom use. J Bisex 2018;18:497-515. 